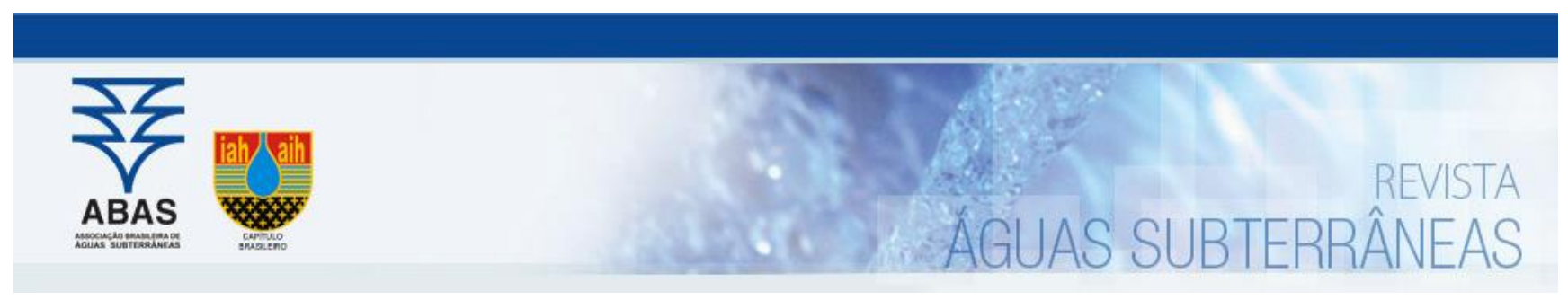

Artigos

\title{
Avaliação de risco ecológico de solos contaminados pelas abordagens ERAGS e TRIAD: aplicabilidade no contexto brasileiro
}

\section{Ecological risk assessment of contaminated soil by the ERAGS and TRIAD approaches: applicability in the brazilian context}

Filipe Viezzer da Silva1; Débora Toledo Ramos; Cristina Cardoso Nunes; Henry Xavier Corseuil1凹

1 Universidade Federal de Santa Catarina (UFSC), Florianópolis, SC

$\bowtie$ filipe.viezzer@ufsc.br,deb.toledo.ramos@gmail.com,c.nunes@ufsc.br, henry.corseuil@ufsc.br

Palavras-chave:

Avaliação de risco ecológico.

ERAGS.

TRIAD.

Solos contaminados.

DD 38/2017.

IN 74/2018.

Keywords:

Ecological risk assessment. ERAGS.

TRIAD.

Contaminated soils.

DD 38/2017.

IN 74/2018.

Revisado por pares.

Recebido em: 15/11/2018.

Aprovado em: 03/05/2019.

Resumo

A constante ameaça aos serviços ecossistêmicos imposta por contaminações antrópicas vem sendo combatida por meio de políticas de gerenciamento de áreas contaminadas. A Avaliação de Risco Ecológico (ARE) tem sido a principal ferramenta de gerenciamento ambiental destas áreas contaminadas, com destaque para as abordagens internacionais ERAGS e TRIAD. No Brasil, a CONAMA 420/2009 prevê a utilização da ARE, porém, atualmente somente as normas estaduais DD 38/2017 (CETESB) e IN 74/2018 (IMA-SC) possuem diretrizes para sua execução. Este trabalho teve como objetivo avaliar a aplicabilidade dessas diretrizes em relação às abordagens ERAGS e TRIAD, bem como avaliar a estrutura normativa existente para realização de testes ecotoxicológicos e ecológicos requisitados em AREs de solos contaminados. Para tanto, foi conduzida uma revisão do quadro normativo internacional da ARE para avaliar a aplicabilidade destas metodologias no contexto brasileiro e um levantamento de 42 avaliações de risco ecológico com abordagens ERAGS e TRIAD, para identificar os testes frequentemente executados e compará-los ao panorama normativo brasileiro. Conclui-se que até o momento (i) há pouco detalhamento nas diretrizes estaduais; (ii) há escassez de infraestrutura laboratorial capacitada para execução dos testes e (iii) há necessidade de uma norma nacional que possa detalhar e viabilizar a execução da ARE no Brasil, a fim de efetivamente promover a proteção dos serviços ecossistêmicos.

Abstract

The constant threats to ecosystem services posed by anthropogenic activities have been managed by contaminatedsites policies. Ecological Risk Assessment (ERA) has been extensively used for the environmental management of contaminated sites, especially through the international approaches - ERAGS and TRIAD. In Brazil, CONAMA $420 / 2009$ suggests the use of ERA, but only the state norms DD 38/2017 (CETESB) and IN 74/2018 (IMA-SC) present guidelines for its execution. The purpose of this review paper was to assess the applicability of these guidelines relative to the ERAGS and TRIAD approaches, as well as to evaluate the existing standard procedures for ecotoxicological and ecological testing required by ERAs of contaminated soils. Accordingly, a review of the international ERA guidelines was conducted to assess the applicability of these methodologies in the Brazilian context, and an assessment of 42 ecological risk assessments that followed ERAGS and TRIAD approaches was used to identify the frequently performed tests and compare them to the Brazilian standard testing panorama. Overall, the current limitations identified were (i) the considerably shallowness of state guidelines; (ii) lack of laboratory infrastructure to perform the tests, which underlines (iii) the need for a national standard guideline that can thoroughly describe the ERA procedures to enable its execution and thereby, effectively promote the protection of ecosystem services.

DOI: http:/dx.doi.org/10.14295/ras.v33i3.29253

\section{INTRODUÇÃO}

A estrutura e as funções ecológicas do solo são responsáveis pela manutenção das condições e dos processos que sustentam a sociedade humana, os chamados serviços ecossistêmicos, tais como o fornecimento do meio de suporte e dos nutrientes para a produção agrícola, o armazenamento e a proteção da água subterrânea, e a ciclagem dos nutrientes (DAILY, 1997; FAO e ITPS, 2015). Estes serviços ecossistêmicos têm sido frequentemente ameaçados pela contaminação antrópica, que se caracteriza como um problema de ocorrência mundial (SWARTJES et al., 2012). Para lidar com os danos aos ecossistemas e à saúde pública, políticas de gerenciamento de áreas contaminadas vêm sendo adotadas em diversos países desde a década de 70, com destaque para o pioneirismo dos EUA e da Holanda (BRANDON, 2013).

De acordo com Nathanail e Bardos (2009), atualmente, as políticas de gerenciamento de áreas contaminadas são 
baseadas em risco e, assim, a tomada de decisão quanto à necessidade de ações de controle e recuperação ambiental é pautada nos resultados de uma avaliação de risco. A Avaliação de Risco Ecológico (ARE) objetiva estimar os efeitos adversos que podem afetar receptores ecológicos, como organismos, populações, comunidades, ecossistemas e etc. (USEPA, 1998). Nas últimas décadas, as AREs de solos contaminados têm sido utilizadas como uma ferramenta de gerenciamento de áreas contaminadas, visando à proteção da biodiversidade e dos serviços ecossistêmicos, devido ao atual estado crítico de degradação do solo (FAO, 2015). Até o momento, a ecotoxicologia do solo ainda é pouco estudada na América Latina, em relação aos ambientes aquáticos (NIEMEYER et al., 2017), havendo, portanto, a necessidade de preencher esta lacuna de conhecimento. A proteção dos ecossistemas terrestres culmina também na preservação de outros recursos de elevada relevância, como por exemplo, a água subterrânea, que se caracteriza como a principal fonte disponível de água para abastecimento.

Dentre os países que possuem longo histórico de gerenciamento de áreas contaminadas, destacam-se as abordagens de ARE dos EUA e da Holanda. A agência de proteção ambiental dos EUA (USEPA) foi pioneira na criação de uma estrutura (USEPA, 1992) e diretrizes para ARE (USEPA, 1998). Em 1997, foi desenvolvido um guia para planejamento e concepção de AREs especificamente para áreas contaminadas do programa Superfund, denominado Ecological Risk Assessment Guidance for Superfund ERAGS (USEPA, 1997). Sua metodologia é amplamente reconhecida e tem sido revisada e adaptada para a aplicação em áreas contaminadas de diversos países (PERRODIN et al., 2011). A avaliação específica do local é denominada Avaliação de Risco Ecológico de Linha de Base (BERA), a qual utiliza uma ou múltiplas linhas de evidência (LoE) para caracterizar o risco. Uma linha de evidência corresponde a um teste que pode indicar dano ecológico real ou potencial. No caso do uso de múltiplas LoE, utiliza-se a ponderação das linhas de evidência baseada no melhor julgamento profissional para a caracterização do risco (USEPA, 1997).

Na Holanda, a Circular de Remediação do Solo (IENM, 2013) define as diretrizes gerais vigentes para ARE em áreas contaminadas, destacando-se a abordagem tríade de qualidade do solo ou TRIAD (JENSEN e MESMAN, 2006) para AREs específicas do local. A TRIAD é derivada da Tríade de Qualidade do Sedimento (LONG e CHAPMAN, 1985) e quantifica o risco por meio da abordagem da ponderação de três linhas de evidências (química, ecotoxicológica e ecológica), através da integração numérica de seus resultados. Esta abordagem é utilizada principalmente em países da Europa e já foi aplicada no Brasil (NIEMEYER et al., 2010, 2015), tendo sido recentemente sistematizada como norma técnica da International Organization for Standardization (ISO, 2017).

No Brasil, as diretrizes para o gerenciamento do solo e das águas subterrâneas contaminadas são estabelecidas na resolução $n^{\circ} 420$, de 2009, do Conselho Nacional de Meio Ambiente (CONAMA, 2009). Esta norma é baseada no modelo holandês de gerenciamento de áreas contaminadas, mas também integra aspectos normativos de outros países, como os EUA (MATIASSO, 2010). A norma nacional tem foco primário no gerenciamento dos riscos à saúde humana, enquanto os riscos ecológicos são abordados de forma secundária. A avaliação de risco ecológico é proposta como uma etapa alternativa, sendo sua efetiva realização dependente da decisão do órgão ambiental competente. Recentemente, a Companhia Ambiental do Estado de São Paulo (CETESB), por meio da Decisão de Diretoria (DD) 38/2017 (CETESB, 2017), tornou a ARE compulsória em locais onde ecossistemas naturais podem ser potencialmente afetados pela contaminação antrópica. Diante desta obrigatoriedade, evidencia-se uma tendência no aumento da exigência, por parte dos órgãos ambientais, da execução de ARE em áreas contaminadas no Brasil, havendo a necessidade de diretrizes orientadoras para nortear sua devida execução.

Neste sentido, a DD 38/2017 define diretrizes gerais e conteúdos a serem abordados em AREs, sem estabelecer uma metodologia específica para sua execução, permitindo, portanto, que o avaliador de risco responsável determine os métodos e abordagens a serem utilizados. As condições de aplicação e diretrizes de ARE estabelecidas pela DD 38/2017 foram adotadas pelo Instituto de Meio Ambiente de Santa Catarina, por meio da Instrução Normativa (IN) 74/2018. Apesar de a Associação Brasileira de Normas Técnicas (ABNT) estar desenvolvendo uma norma técnica nacional para ARE, que deverá detalhar os procedimentos já requeridos pelas normas estaduais, a DD 38/2017 e IN $74 / 2018$ se constituem como as únicas diretrizes orientadoras atualmente disponíveis no Brasil.

Como a DD 38/2017 e a IN 74/2018 não possuem bases metodológicas para balizar a execução da ARE e são baseadas em orientações internacionais, este trabalho objetiva avaliar a aplicabilidade das normas internacionais em relação à conformidade e estrutura normativa das abordagens ERAGS e TRIAD, bem como aos testes ecotoxicológicos e ecológicos comumente requisitados em AREs de solos contaminados.

\section{MATERIAIS E MÉTODOS}

Este artigo contempla uma revisão bibliográfica do quadro normativo nacional e internacional, relacionados à avaliação de risco ecológico de solos contaminados e das abordagens ERAGS (USEPA, 1997) e TRIAD (JENSEN e MESMAN, 2006) para avaliação específica do local, visando analisar a aplicabilidade destas metodologias no contexto brasileiro, por meio das diretrizes disponíveis atualmente no Brasil (DD 38/2017 e IN 74/2018).

Neste escopo, foram apresentados os testes ecotoxicológicos e ecológicos recomendados para execução de AREs e comparados ao panorama das normas técnicas e protocolos brasileiros existentes para a condução destes testes. Estudos de caso que utilizaram abordagens ERAGS e TRIAD foram avaliados para identificar os testes mais frequentemente executados e para verificar eventuais convergências entre os testes conduzidos em ambas as abordagens. Dentre os estudos de caso, aqueles que utilizaram a abordagem 
ERAGS foram pesquisados na base de dados pública de áreas contaminadas do Programa Superfund da USEPA (https://cumulis.epa.gov/supercpad/cursites/srchsites.cfm), enquanto que periódicos e outras publicações científicas foram utilizados para a pesquisa dos estudos de caso da TRIAD. Ao contrário da USEPA, o Instituto Holandês para a Saúde Pública e Meio Ambiente (sigla do holandês, RIVM), não possui uma base de dados pública de AREs e, por isso, a busca se restringiu a documentos científicos. Estes estudos de caso totalizaram 42 avaliações de risco ecológico, sendo 27 provenientes da ERAGS e 15 da TRIAD. As normas técnicas e protocolos brasileiros foram buscados na base de dados da Associação Brasileira de Normas Técnicas (ABNT), da Empresa Brasileira de Pesquisa Agropecuária (Embrapa) e da Sociedade Brasileira de Ciência do Solo (SBCS).

\section{RESULTADOS E DISCUSSÃO}

\subsection{Conformidade das abordagens ERAGS e TRIAD aos requisitos da DD 38/2017 e IN 74/2018}

A DD 38/2017 e a IN 74/2018 orientam a ARE por meio da definição dos conteúdos a serem abordados no modelo conceitual, na metodologia de avaliação de risco e na interpretação dos resultados da ARE. Estes conteúdos são, em sua maioria, etapas gerais de uma avaliação de risco, e também orientam a forma de execução das etapas (e.g. mínimo de três linhas de evidência). Assim, para avaliar a aplicabilidade das abordagens ERAGS e TRIAD, no contexto da DD 38/2017 e IN 74/2018, foi verificado se os requisitos destas diretrizes estão previstos (destacados em verde) ou não (destacados em vermelho) nas normas internacionais, no que se refere à metodologia e à interpretação dos resultados da avaliação de risco (Quadro 1).

Quadro 1 - Conformidade das abordagens ERAGS e TRIAD aos requisitos da DD 38 (CETESB, 2017) e IN 74 (IMA, 2018)

\begin{tabular}{|c|c|c|}
\hline Diretrizes da DD 38/IN 74 & ERAGS & TRIAD \\
\hline \multicolumn{3}{|l|}{ A) Conteúdo metodológico da Avaliação de Risco Ecológico: } \\
\hline \multicolumn{3}{|l|}{ i. Descrição e justificativa da metodologia selecionada; } \\
\hline \multicolumn{3}{|l|}{$\begin{array}{l}\text { ii. Apresentação das linhas de evidências, considerando no mínimo três linhas: química, } \\
\text { ecotoxicológica e ecológica; }\end{array}$} \\
\hline \multicolumn{3}{|l|}{ iii. Descrição de incertezas analíticas e de modelos; } \\
\hline \multicolumn{3}{|l|}{$\begin{array}{l}\text { iv. Apresentação dos critérios de avaliação para cada linha de evidência e por compartimen- } \\
\text { to ambiental; }\end{array}$} \\
\hline \multicolumn{3}{|l|}{ v. Apresentação da base dos cálculos de risco, informando o nível de risco aceitável; } \\
\hline \multicolumn{3}{|l|}{$\begin{array}{l}\text { vi. Identificação de área de referência com características semelhantes à área contaminada, } \\
\text { por compartimentos ambientais; }\end{array}$} \\
\hline \multicolumn{3}{|l|}{ vii. Descrição e localização dos pontos de coleta por Unidade de Exposição; } \\
\hline \multicolumn{3}{|l|}{ viii. Descrição e justificativa das metodologias de amostragem e de ensaios. } \\
\hline \multicolumn{3}{|l|}{ B) Conteúdo interpretativo dos resultados da avaliação de risco: } \\
\hline \multicolumn{3}{|l|}{$\begin{array}{l}\text { i. A quantificação do risco para cada substância química de interesse, em cada caminho de } \\
\text { exposição considerado em cada unidade de exposição; }\end{array}$} \\
\hline \multicolumn{3}{|l|}{ ii. A quantificação do risco total para cada unidade de exposição; } \\
\hline \multicolumn{3}{|l|}{$\begin{array}{l}\text { iii. O cálculo das Concentrações Máximas Aceitáveis (CMA) para cada substância química de } \\
\text { interesse em cada compartimento do meio, por unidade de exposição; }\end{array}$} \\
\hline \multicolumn{3}{|l|}{ iv. Mapas de risco com a indicação dos receptores e os hot spots; } \\
\hline v. A conclusão acerca da necessidade de implementação de medidas de intervenção. & & \\
\hline
\end{tabular}

A maioria dos conteúdos requeridos pela DD 38/2017 e IN 74/2018 está prevista nas abordagens ERAGS e TRIAD, que atendem a 9 dos 13 requisitos. Contudo, algumas divergências foram identificadas. Em relação ao conteúdo metodológico da avaliação de risco ecológico (A), o item a A-i foi considerado como previsto por serem abordagens reconhecidas internacionalmente e por serem orientadoras de AREs de áreas contaminadas nos EUA e na Holanda. No item A-ii, a abordagem ERAGS não define um número mínimo de linhas de evidência a ser considerado na ARE, destacando que a comparação da concentração ambiental com um valor toxicológico de referência, característico da LoE química, pode ser a única LoE necessária ou viável. Já a TRIAD realiza, fundamentalmente, a avaliação combinada de três linhas de evidência: química, ecotoxicológica e ecológica, estando, portanto, em convergência com as exigências das diretrizes nacionais. No que diz respeito à descrição das incertezas, item A-iii, a ERAGS exige uma análise de incertezas, já a TRIAD não prevê essa etapa, mas calcula o desvio entre os resultados das diferentes LoEs. 0 desvio indica a incerteza no resultado representando a convergência (ou divergência) nos resultados de cada LoE. A respeito dos itens A-iv e A-viii, ambos previstos pelas duas abordagens, os critérios de avaliação de cada LoE, assim como as metodologias de ensaios e amostragem a serem aplicadas, devem estar relacionados aos aspectos ecológicos a serem protegidos (SWARTJES et al., 2011). Enquanto a abordagem ERAGS não oferece orientações explícitas em relação aos critérios de avaliação e aos ensaios a serem realizados, a TRIAD sugere ensaios específicos, relacionando sua relevância com o uso do solo pretendido na área de estudo (JENSEN e MESMAN, 2006; MESMAN et al., 2014). 
Para o item A-v, novamente a ERAGS propõe formulações para o cálculo do risco ou limites para o risco aceitável. Por outro lado, a TRIAD apresenta métodos de cálculo para diversas linhas de evidência e indica o uso de uma matriz de decisão para determinação do risco. Esta matriz define intervalos de valores de risco que integram os resultados das diferentes linhas de evidência e seus respectivos valores de desvio, classificando o risco como aceitável ou não aceitável para diferentes usos do solo, conforme exemplificado em Jensen e Mesman (2006). Em relação ao item A-vi, ambas as abordagens possuem conformidades com a DD 38 e IN 74, já que a seleção de uma área de referência, sem contaminação, tem o propósito de ser utilizada para a comparação e normalização dos resultados de análises da área contaminada (SUTER et al., 2000). 0 solo da área de referência e da área contaminada devem assemelhar-se em determinados parâmetros, como textura (frações de argila, silte e areia), pH, teor de matéria orgânica, capacidade de retenção de água e teor de nutrientes (JENSEN e MESMAN, 2006), uma vez que estes parâmetros exercem influência sobre a biodisponibilidade dos contaminantes (NAIDU et al., 2008) e a composição e atividade da biota do solo (BARDGETT, 2005). Sobre o item A-vii, as abordagens ERAGS e TRIAD não definem explicitamente métodos para determinar pontos de coleta, porém, sugerem que a distribuição dos pontos de coleta represente o gradiente de contaminação. Segundo USEPA (1997), pontos de coleta que representem um gradiente de efeitos ao longo de diferentes níveis de contaminação possibilitam o estabelecimento de causalidade e proporcionam uma avaliação da relação exposição-resposta que auxilia a identificação das CMAs (concentrações máximas aceitáveis, acima das quais há risco não aceitável e requer medidas de intervenção).

Quanto ao conteúdo interpretativo dos resultados da avaliação de risco (B), o item B-i não é atendido pela TRIAD, uma vez que esta se propõe a avaliar o risco relacionado à mistura de contaminantes e, na LoE química, o risco é calculado para cada substância e, subsequentemente, integrado em um único valor. Assim, seria possível o cálculo do risco individual de cada substância, apesar de esta abordagem estar pautada no risco imposto pela mistura de contaminantes. Já para as LoEs ecotoxicológicas e ecológicas, não se avalia o efeito específico de cada contaminante da mistura, para ambas abordagens. Para a quantificação do risco total, item B-ii, a ERAGS prevê o uso de uma abordagem de ponderação de evidências baseada no melhor julgamento profissional para a caracterização do risco de múltiplas LoE e não propõe um método quantitativo para a integração dos resultados, conforme exigido pelas diretrizes nacionais. Por outro lado, a TRIAD traz uma abordagem quantitativa de ponderação de evidência, onde os resultados de cada LoE são escalonados, ponderados e integrados matematicamente. Nos itens B-iii e B-iv, verifica-se que a TRIAD não prevê a derivação das CMAs e a apresentação de mapas de risco como resultado da avaliação. Já a ERAGS indica a representação espacial da extensão da contaminação acima de um limiar de efeito inaceitável (valores acima da (MA), mas não especifica a representação do risco por receptor e a localização de hot spots. 0 item B-v, que dispõe sobre a conclusão acerca da necessidade de implementação de medidas de intervenção, é abordado de maneira diferente nas metodologias ERAGS e TRIAD.

Ambas apontam que os resultados da avaliação de risco de vem subsidiar a etapa de gerenciamento de risco, onde a decisão sobre as ações de controle do risco e redução do risco devem ser definidas. Adicionalmente, a ERAGS indica que a decisão sobre as formas de intervenção devem, entre outros aspectos, levar em consideração os impactos e os riscos ecológicos das diferentes estratégias de remediação, incluindo a alternativa de não intervenção.

De maneira geral, a abordagem ERAGS diverge da DD $38 / 2017$ e da IN 74/2018 por não exigir o uso mínimo de 3 LoEs (A-ii), não propor uma base de cálculo do risco, nem limites de risco aceitável (A-v), não prever a quantificação do risco total (B-ii) e não exigir mapas de risco (B-iv). A ERAGS deixa a cargo do gestor e do avaliador de risco a escolha dos métodos a serem utilizados em cada etapa. Por outro lado, a TRIAD converge em mais itens previstos como conteúdo metodológico ( 7 de 8 itens) e diverge em mais itens do conteúdo interpretativo (3 de 5 itens). Enfim, a abordagem TRIAD não prevê explicitamente uma descrição de incertezas (A-iii), não prevê o cálculo do risco para cada substância química (B-i) e da CMA (B-iii), e não indica a apresentação de mapas de risco (B-iv). Na TRIAD há a proposição de métodos específicos a serem utilizados nas etapas, porém, não aborda todos os conteúdos requeridos pelas normas estaduais.

Em última análise, foram evidenciadas inconformidades em ambas as abordagens ERAGS e TRIAD em relação à DD 38/2017 e IN 74/2018. Diante disso, na ausência de uma norma nacional, a realização das AREs no Brasil dependerá do diálogo entre os órgãos fiscalizadores e os avaliadores de risco para esclarecimentos e orientações em relação às lacunas que foram identificadas, visando o cumprimento dos requisitos das diretrizes estaduais existentes.

\subsection{Panorama das normas e protocolocos brasileiros para testes ecotoxicológicos e ecológicos utilizáveis em AREs}

Para avaliação de AREs são necessários testes químicos, ecotoxicológicos e ecológicos. Os testes da LoE química não foram aqui abordados, pois apresentaram pouca variação e por ter sido predominantemente utilizada a determinação da concentração total das substâncias em solo, a qual possui protocolos bastante consolidados no Brasil. Assim, são destacados nestes resultados apenas os testes das LoEs ecotoxicológica e ecológica mais utilizados nos estudos de caso da ERAGS e da TRIAD, e discutidas as respectivas normas técnicas e protocolos nacionais para sua execução.

A escolha das linhas de evidência é uma etapa crucial da ARE específica do local e deve ser orientada pelos aspectos 
ecológicos a serem protegidos, pelas substâncias químicas de interesse e pelas características ecológicas da área em estudo. A TRIAD propõe testes a serem realizados, destacando sua relevância em relação ao uso do solo pretendido na área de estudo, enquanto que a ERAGS não apresenta recomendações de testes específicos para a ARE de solo contaminado. 0 Quadro 2 apresenta a relação dos principais testes realizados em AREs, segundo as aborda- gens ERAGS e TRIAD, com seus protocolos de execução nacionais e internacionais associados. Os testes foram ainda classificados de acordo com sua frequência de utilização (alta, média e baixa) nos 42 estudos de caso, e com a convergência entre as abordagens. Dentre os testes com frequência baixa, foram apresentados somente aqueles que possuem protocolo nacional.

Quadro 2 - Relação dos principais testes das linhas de evidência ecotoxicológica e ecológica, segundo estudos das abordagens ERAGS e TRIAD, e suas respectivas normas técnicas e protocolos de execução (testes sublinhados indicam convergência entre as abordagens ERAGS e TRIAD)

\begin{tabular}{|c|c|c|}
\hline LoE (teste) & Protocolo nacional & Protocolo internacional1 \\
\hline \multicolumn{3}{|l|}{ LoE Ecotoxicológica } \\
\hline $\begin{array}{l}\text { Plantas: emergência de sementes ou cresci- } \\
\text { mento }\end{array}$ & ABNT NBR ISO 11269-2 (2014) & - \\
\hline Minhocas: reprodução & - & ISO 11268-2 (2012) \\
\hline Minhocas: letalidade, crescimento & ABNT NBR ISO 15537 (2014) & - \\
\hline $\begin{array}{l}\text { V. fischeri2: inibição de luminescência (Micro- } \\
\text { tox) }\end{array}$ & ABNT NBR 15411-3 (2012) & - \\
\hline Enquitreídeos: reprodução & ABNT NBR ISO 16387 (2012) & - \\
\hline Colêmbolos: reprodução & ABNT NBR ISO 11267 (2011) & - \\
\hline Ostracodos 2 : letalidade, crescimento & - & ISO 14371 (2012) \\
\hline Dafnídeos2: letalidade & ABNT NBR 12713 (2016) & - \\
\hline Biomarcadores: plantas, minhocas e ameba & - & $\begin{array}{l}\text { Semenzin et al. (2008), Gutierrez } \\
\text { et al. (2015) }\end{array}$ \\
\hline Minhocas: fuga & ABNT NBR ISO 17512-1 (2011) & - \\
\hline Dafnídeos²: reprodução & ABNT NBR 13373 (2017) & - \\
\hline Microalgas²: viabilidade & ABNT NBR 12648 (2018) & - \\
\hline \multicolumn{3}{|l|}{ LoE Ecológica } \\
\hline Levantamento dos invertebrados & $\begin{array}{l}\text { Aquino et al. }(2006)^{3} \\
\text { Dionísio et al. }(2016)^{3}\end{array}$ & - \\
\hline Levantamento dos nematoides & Dionísio et al. $(2016)^{3}$ & - \\
\hline Microrganismos: mineralização do C e N & - & $\begin{array}{l}\text { ISO } 14238 \text { (2012), } \\
\text { ISO } 15685 \text { (2012) }\end{array}$ \\
\hline Teste de respiração basal do solo (RBS) & $\begin{array}{l}\text { Silva et al. (2007c), } \\
\text { Dionísio et al. (2016) }\end{array}$ & - \\
\hline Microrganismos: número e biomassa & Silva et al. $(2007 a, b)$ & - \\
\hline Microrganismos: atividade enzimática & - & ISO 23753-1 (2005) \\
\hline $\begin{array}{l}\text { Microrganismos: diversidade metabólica (Bio- } \\
\text { log) }\end{array}$ & - & Insam (1997) \\
\hline $\begin{array}{l}\text { Teste de respiração induzida por substrato } \\
\text { (RIS), }\end{array}$ & Dionísio et al. (2016) & - \\
\hline Decomposição (método litter bag) & Dionísio et al. (2016) & - \\
\hline Microrganismos: diversidade genética & Aboim et al. (2004) & - \\
\hline \multicolumn{3}{|c|}{ 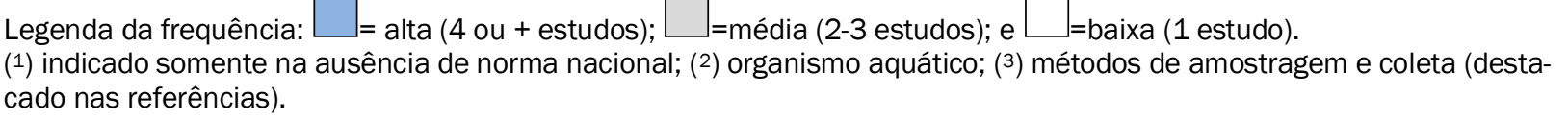 } \\
\hline
\end{tabular}

Dentro da linha de evidência ecotoxicológica, os bioensaios de letalidade e de crescimento com minhocas, e os bioensaios de emergência de semente e de crescimento de plantas foram os mais frequentes e utilizados por ambas as abordagens. Na TRIAD foram conduzidos bioensaios com organismos aquáticos para avaliação da qualidade do solo.
Niemeyer et al. (2015) destacaram sua utilidade na avaliação da função de retenção do solo, estimando, assim, os efeitos ecológicos potenciais da contaminação da água subterrânea e superficial. 0 bioensaio aquático mais recorrente foi o teste microtox, de inibição de luminescência em bactérias ( $V$. fischeri). A maioria dos estudos TRIAD 
utilizou organismos-teste de diferentes níveis tróficos (plantas, invertebrados e microrganismos) e funcionais (decomposisção da matéria orgânica e ciclagem de nitrientes), e a avaliação de efeitos agudos e crônicos, o que fornece um entendimento mais robusto sobre a biodisponibilidade dos contaminantes e de seus impactos potenciais ao ecossistema terrestre.

Os testes da linha de evidência ecológica que convergiram em ambas as abordagens se referem aos levantamentos quantitativos da riqueza e diversidade da comunidade dos invertebrados. Somente as AREs que utilizaram a abordagens ERAGS apresentaram levantamentos observacionais qualitativos dos efeitos negativos da contaminação em plantas, tais como: presença de vegetação estressada, raquítica ou com crescimento anormal; redução da diversidade da comunidade; e a presença de espécies tolerantes à contaminação. As demais LoEs ecológicas utilizadas na TRIAD apresentaram somente análises quantitativas, com destaque para levantamentos de nematoides e microartrópodos, testes com microrganismos e medição da atividade de invertebrados do solo. De uma forma geral, a ERAGS focou suas avaliações nos grupos dos invertebrados e das plantas, enquanto que a TRIAD, analisou também microrganismos e aspectos ecológicos funcionais relacionados à ciclagem dos nutrientes e à decomposição da matéria orgâ- nica (litter bags).

A maioria das LoEs ecotoxicológicas utilizadas nos estudos ERAGS e TRIAD possui normas técnicas e protocolos nacionais para sua execução (Quadro 3). As normas brasileiras para ensaios ecotoxicológicos são baseadas em reconhecidos protocolos da ISO, ASTM (Sociedade Americana de Testes e Materiais) e OECD (Organização para a Cooperação e Desenvolvimento Econômico). Isso indica que, do ponto de vista normativo, não haveria dificuldades em utilizar linhas de evidências ecotoxicológicas em AREs a serem realizadas no Brasil.

A viabilidade de execução dos testes ecotoxicológicos e ecológicos também é dependente da disponibilidade de instituições credenciadas que possuam capacidade técnica para realizá-los, não bastando apenas a existência de normas técnicas para sua execução. Diante disso, Niva et al. (2016) avaliaram a infraestrutura de laboratórios no Brasil que realizam testes ecotoxicológicos e ecológicos. Estas informações foram sintetizadas no Quadro 3, onde as instituições que realizam os testes estão separadas por região e por frequência (ativa, esporadicamente e descontínua) e tipo de análise (plantas, invertebrados e microrganismos), desenvolvidas nos 42 estudos de caso analisados.

Quadro 3 - Instituições brasileiras ativas (vermelho), esporadicamente ativas (azul) ou com atividades descontinuadas (verde) em ecotoxicologia do solo

\begin{tabular}{|c|c|c|c|}
\hline Instituição & Tipo de análise & Instituição & Tipo de análise \\
\hline \multicolumn{4}{|l|}{ Região Sudeste } \\
\hline Aplysia* (ES) & $\mathrm{ML} \mathrm{P}$ & Instituto Biológico (SP) & MLRFB m \\
\hline CENA-USP (SP) & $\mathrm{m}$ & Solotox* (RJ) & MLRF ELR \\
\hline CETEM (RJ) & MLB $\mathrm{m}$ & UFMG (MG) & MLRF \\
\hline EESC-USP (SP) & MLRF CLRF ELR & UFES (ES) & $M \underline{L}$ \\
\hline Embrapa Agrobiologia (RJ) & MLRF ELR & UFF (RJ) & MLRF CLRF ELR \\
\hline ESALQ (SP) & MLRF CLRF $\mathrm{m}$ & UNICAMP (SP) & M \\
\hline IAC (SP) & $\mathrm{P}$ & & \\
\hline \multicolumn{4}{|l|}{ Região Sul } \\
\hline Bioensaios* (RS) & $M L \mathrm{~m}$ & UFFS (SC) & MLRF CLRF ELR \\
\hline Embrapa Florestas (PR) & $\mathrm{MLF}$ & UFPR (PR) & MLF \\
\hline FEPAM (RS) & $\mathrm{P}$ & UFSC (SC) & MLRF CLRF ELR \\
\hline FURG (RS) & $\mathrm{P}$ & UFSM (RS) & $M \underline{L}$ \\
\hline UDESC (SC) & MLRF CLRF ELR $\mathrm{m}$ & Universidade Positivo (PR) & MLF \\
\hline UEL (PR) & MLF & UTFPR (PR) & ELR \\
\hline \multicolumn{4}{|c|}{ Região Centro-Oeste, Nordeste e Norte } \\
\hline Embrapa Cerrados (DF) & ELR & UFPE (PE) & M \\
\hline UFBA (BA) & MLRF CLRF ELR & Embrapa Amazônia (AM) & MLRF \\
\hline $\begin{array}{l}\text { Legenda dos testes ecotoxicoló } \\
\text { m=microrganismos; } P=\text { plantas; } \\
\text { (*) Laboratórios comerciais. }\end{array}$ & $\begin{array}{l}\text { cos e/ou ecológicos: } \\
=\text { letalidade; } \mathrm{R}=\text { letalid }\end{array}$ & $\begin{array}{l}\text { nhocas; } C=\text { colêmbolos; } E=e \\
E=\text { fuga; e } \underline{B}=\text { bioacumulação }\end{array}$ & \\
\hline
\end{tabular}

Fonte: modificado de Niva et al. (2016).

As regiões sudeste e sul concentram o maior número de instituições ativas e conduzem os testes mais frequentes em AREs. Quanto aos testes disponíveis, verifica-se que os bioensaios com minhocas, enquitreídeos e/ou colêmbolos são realizados em um maior número de instituições, enquanto que ensaios com plantas e microrganismos são aparentemente menos frequentes. Além disso, destaca-se que apenas três instituições são laboratórios privados que prestam serviço ao público. Por outro lado, nas regiões centro-oeste, nordeste e norte, há apenas três instituições ativas e não estão disponíveis testes com plantas. Este cenário de escassez de instituições disponíveis para reali- 
zação dos ensaios ecotoxicológicos e ecológicos poderá dificultar a logística de transporte de amostras para os laboratórios e prejudicar a execução de AREs em solos contaminados no Brasil, especialmente nas regiões centrooeste, nordeste e norte, onde há uma maior carência de instituições capazes de conduzir os ensaios.

Portanto, apesar de existir uma estrutura normativa satisfatória para os testes ecotoxicológicos, há escassez de instituições para executá-los, com limitações acentuadas nas regiões norte, nordeste e centro-oeste, podendo dificultar a execução das AREs no Brasil. Em relação aos testes ecológicos, há menor disponibilidade de protocolos padronizados e também há limitações em relação ao número de instituições capacitadas para conduzir estes testes, o que pode dificultar ainda mais a sua realização.

\section{CONCLUSÃO}

A maioria dos conteúdos requeridos pela DD 38/2017 e IN 74/2018 estão previstos nas abordagens ERAGS e TRIAD. As divergências apresentadas pela ERAGS estão relacionadas ao fato de não haver indicação explícita de formas de cálculo de risco, níveis de risco aceitável e metodologia para quantificação do risco total. Com relação à TRIAD, as principais divergências se referem aos conteúdos interpretativos dos resultados, os quais estão limitados à caracterização do risco, sem apresentar aspectos ligados à fase de gerenciamento de risco ou de intervenção. Diante disso, a realização das AREs no Brasil dependerá do diálogo entre os órgãos fiscalizadores e os avaliadores de risco para esclarecimentos e orientações, para permitir o seu cumprimento.

A estrutura de normas técnicas para os testes ecotoxicologicos se mostrou satisfatória, já para os testes ecológicos, há menor disponibilidade de protocolos nacionais. Apesar disso, há escassez de infraestrutura de laboratórios capacitados para a execução desses testes. No Brasil, como um todo, somente 29 laboratórios poderiam prestar serviços de testes ecotoxicológicos e ecológicos, caracterizando uma carência de instituições. Essas instituições são, em sua maioria, de pesquisa, e estão concentradas nas regiões sul (12) e sudeste (13), o que indica que a logística de transporte de amostras para os laboratórios competentes pode representar um fator limitante para a execução de AREs no Brasil, especialmente se os casos de contaminação ocorreram nas regiões norte, nordeste ou centro-oeste.

Conclui-se, portanto, que as limitações para execução de ARE até o momento referem-se à (i) falta de detalhamento das diretrizes estaduais; (ii) à escassez de infraestrutura laboratorial capacitada para execução dos testes e (iii) à necessidade de uma norma nacional que possa detalhar os requisitos já definidos pelas normativas pioneiras. E, diante disso, destaca-se a importância da publicação da norma nacional de ARE, atualmente em desenvolvimento pela ABNT, que possa detalhar e aperfeiçoar os requisitos já definidos pelas normativas pioneiras, DD 38/2017 e IN
$74 / 2018$, indicando os testes a serem executados. Além disso, ressalta-se a necessidade do estabelecimento de instituições capacitadas para executar os testes comumente requisitados pelas AREs, em todas as regiões do país, a fim de viabilizar sua execução no Brasil e, consequentemente, de efetivamente promover a proteção dos serviços ecossistêmicos.

\section{AGRADECIMENTOS}

Este trabalho contou com apoio da CAPES, Coordenação de Aperfeiçoamento de Pessoal de Nível Superior - Brasil.

\section{REFERÊNCIAS}

ABOIM, M.C.R.; BARBOSA, J.C.; COUTINHO, H.L.C.; ROSADO, A.S. Avaliação da diversidade microbiana em amostras de solos: Técnica do PCR/DGGE (Protocolo Laboratorial). Documentos 68. Rio de Janeiro: Embrapa Solos, 2004.

ABNT - Associação Brasileira de Normas Técnicas. NBR ISO 11267: Qualidade do solo - Inibição da reprodução de Collembola (Folsomia candida) por poluentes do solo. Rio de janeiro, 2011.

. NBR ISO 17512-1: Qualidade do Solo - Ensaio de fuga para avaliar a qualidade de solos e efeitos de substâncias químicas no comportamento Parte 1: Ensaio com minhocas (Eisenia fetida e Eisenia andrei). Rio de janeiro, 2011.

NBR 15411-3: Ecotoxicologia aquática - Determinação do efeito inibitório de amostras aquosas sobre a emissão da bioluminescência de Vibrio fischeri (ensaio de bactéria luminescente). Parte 3: Método utilizando bactérias liofilizadas. Rio de Janeiro, 2012.

NBR ISO 16387: Qualidade do solo - Efeitos de poluentes em Enchytraeidae (Enchytraeus sp.) - Determinação de efeitos sobre reprodução e sobrevivência. Rio de Janeiro, 2012.

. NBR ISO 11269-2: Qualidade do solo - Determinação dos efeitos de poluentes na flora terrestre - Parte 2 Efeitos do solo na emergência e no crescimento de vegetais superiores. Rio de Janeiro, 2014.

NBR ISO 15537: Ecotoxicologia terrestre - Toxicidade aguda - Método de ensaio com minhocas (Lumbricidae). Rio de Janeiro, 2014.

NBR 12713: Ecotoxicologia aquática - Toxicidade aguda - Método de ensaio com Daphnia spp (Crustacea, Cladocera). Rio de Janeiro, 2016.

. NBR 13373: Ecotoxicologia aquática - Toxicidade crônica - Método de ensaio com Ceriodaphnia spp (Crustacea, Cladocera). Rio de Janeiro, 2017.

AQUINO, A.M.; AGUIAR-MENEZES, E.L.; QUEIROZ, J.M. Re- 
comendações para coleta de artrópodes terrestres por armadilhas de queda ("pitfall-traps"). Circular Técnica 18. Seropédica: Embrapa Agrobiologia, 2006.

BARDGETT, R.D. The biology of soil: a community and ecosystem approach. Oxford, UK: Oxford University Press, 2005.

BRANDON, E. Global approaches to site contamination law. Springer, 2013.

CETESB - Companhia Ambiental do Estado de São Paulo. Decisão de Diretoria $N^{\circ}$ 038/2017/C, de 07 Fevereiro de 2017. Dispõe sobre a aprovação do "Procedimento para a Proteção da Qualidade do Solo e das Águas Subterrâneas", da revisão do "Procedimento para o Gerenciamento de Áreas Contaminadas" e estabelece "Diretrizes para Gerenciamento de Áreas Contaminadas no Âmbito do Licenciamento Ambiental", em função da publicação da Lei Estadual $n^{\circ} 13.577 / 2009$ e seu Regulamento, aprovado por meio do Decreto $n^{\circ} 59.263 / 2013$, e dá outras providências. São Paulo, 2017.

CONAMA - Conselho Nacional de Meio Ambiente. Resolução $n^{\circ}$ 420, de 28 de Dezembro de 2009. Dispõe sobre critérios e valores orientadores de qualidade do solo quanto à presença de substâncias químicas e estabelece diretrizes para o gerenciamento ambiental de áreas contaminadas por essas substâncias em decorrência de atividades antrópicas. Diário Oficial da União n² 249, de 30 dez. 2009.

DAILY, G. C. What Are Ecosystem Services? In: DAILY, G. C. (Ed.). Nature's services: societal dependence on natural ecosystems. Washington, DC: Island Press, 1997.

DIONÍSIO, J.A.; PIMENETEL, I.C.; SIGNOR, D.; PAULA, A.M.; MACEDA, A.; MATTANA, A.L. Guia prático de biologia do solo. Curitiba, PR: SBCS, 2016. 152 p.

FAO - Food and Agriculture Organization of the United Nations. Soil and Pulses - Simbiosis for Life. Rome, IT: FAO, 2016.

FAO - Food and Agriculture Organization of the United Nations; ITPS - Intergovernmental Technical Panel on Soils. Status of the World's Soil Resources (SWSR) - Main Report. Rome, IT: FAO, 2015.

GUTIÉRREZ, L.; GARBISU, C.; CIPRIÁN, E.; BECERRIL, J.M.; SOTO, M.; ETXEBARRIA, J.; MADARIAGA, J.M.; ANTIGÜEDAD, I.; EPELDE, L. Application of ecological risk assessment based on a novel TRIAD-tiered approach to contaminated soil surrounding a closed non-sealed landfill. Science of the Total Environment, vol. 514, p.49-59, 2015.

IENM - Ministry of Infrastructure and the Environment. Soil Remediation Circular 2013, version of 1 July 2013.
INSAM, H. A new set of substrates proposed for community characterization in environmental samples. In: Insam, H., Rangger, A. (Eds.), Microbial Communities. Functional Versus Structural Approaches. Heidelber: Springer, 1997.

IMA - Instituto do Meio Ambiente do Estado de Santa Catarina. Instrução Normativa $n^{\circ} 74$, de 29 de agosto de 2018. Recuperação de Áreas Contaminadas. Florianópolis, 2018.

ISO - International Organization for Standardization. ISO 23753-1: Soil quality - Determination of dehydrogenase activity in soils - Part 1: Method using triphenyltetrazolium chloride (TTC). Switzerland, 2012.

ISO 11268-2: Soil quality - Effects of pollutants on earthworms - Part 2: Determination of effects on reproduction of Eisenia fetida/Eisenia Andrei. Switzerland, 2012.

ISO 14238: Soil quality - Biological methods - Determination of nitrogen mineralization and nitrification in soils and the influence of chemicals on these processes. Switzerland, 2012.

ISO 15685: Soil quality - Determination of potential nitrification and inhibition of nitrification - Rapid test by ammonium oxidation. Switzerland, 2012.

ISO 14371: Water quality - Determination of fresh water sediment toxicity to Heterocypris incongruens (Crustacea, Ostracoda). Switzerland, 2012.

ISO 19204:2017: Soil quality - Procedure for sitespecific ecological risk assessment of soil contamination (soil quality TRIAD approach). Switzerland, 2017.

JENSEN, J.; MESMAN, M. (Eds.). Ecological risk assessment of contaminated land: Decision support for specific investigations. RIVM report number 711701047, 2006.

LONG, E.R.; CHAPMAN, P.M. A Sediment Quality Triad: Measures of Sediment Contamination, Toxicity and Infaunal Community Composition in Puget Sound. Marine Pollution Bulletin, v. 16, n. 10, p. 405-415, 1985.

MATIASSO, D. Nova fase para gestão de solos contaminados. Revista água e meio ambiente, n. 14, ano 3, p. 18-19, fevereiro/março 2010.

MESMAN, M.; RUTGERS, M.; SCHOUTEN, A.J.; BOGTE, J.J.; DIRVEN-VAN BREEMEN, E.M. Evaluatie van Triadeonderzoeken op schietterreinen van defensie. RIVM Rapport 2014-0077, 2014.

NAIDU, R.; BOLAN, N.S.; MEGHARAJ, M.; JUHASZ, A.L.; GUPTA, S.K.; CLOTHIER, B.E.; SCHULIN, R. Chemical bioavailability in terrestrial environments. In: NAIDU, R. (Ed.). Chemical Bioavailability in Terrestrial Environments. Developments in Soil Science - Volume 32. Amsterdam, NL: Elsevier B.V., 2008. 
NATHANAIL, C.P.; BARDOS, R.P. Reclamation of Contaminated Land. Chichester, UK: John Wiley \& Sons Ltd., 2004.

NIEMEYER, J.C.; MOREIRA-SANTOS, M.; NOGUEIRA, M.A.; CARVALHO, G.M.; RIBEIRO, R.; SILVA, E.M.; SOUSA, J.P. Environmental risk assessment of a metal-contaminated area in the Tropics. Tier I: screening phase. Journal of Soils and Sediments, v. 10, p. 1557-1571, 2010.

NIEMEYER, J.C.; CHELINHO, S.; SOUSA, J.P. soil ecotoxicology in Latin America: current research and perspectives. Environmental Toxicology and Chemistry, v. 36, n. 7, p. 1795-1810, 2017.

NIEMEYER, J.C.; MOREIRA-SANTOS, M.; RIBEIRO, R.; RUTGERS, M.; NOGUEIRA, M.A.; SILVA, E.M.; SOUSA, J.P. Ecological Risk Assessment of a Metal Contaminated Area in the Tropics. Tier II: Detailed Assessment. Plos One, v. 10, n. 11, 2015.

NIVA, C.C.; NIEMEYER, J.C.; SILVA, F.M.R.J.; NUNES, M.E.T.; SOUSA, D.L.; ARAGÃO, C.W.S.; SAUTTER, K.D.; ESPINDOLA, E.G.; SOUSA, J.P.; RÖMBKE, J. Soil ecotoxicology in Brazil is taking its course. Environmental Science and Pollution Research, v. 23, n. 11, p 11363-11378, 2016.

PERRODIN, Y.; BOILLOT, C.; ANGERVILLE, R.; DONGUY, G.; EMMANUEL, E. Ecological risk assessment of urban and industrial systems: A review. Science of the Total Environment, v. 409, p. 5162-5176, 2011.

SEMENZIN, E.; CRITTO, A.; RUTGERS, M. MARCOMINI, A. Integration of bioavailability, ecology and ecotoxicology by three lines of evidence into ecological risk indexes for contaminated soil assessment. Science of the Total Environment, v. 389, p. 71-86, 2008.

SILVA, E.E.; AZEVEDO, P.H.S.; DE-POLLI, H. Determinação do nitrogênio da biomassa microbiana do solo (BMS-N). Comunicada Técnico 96. Seropédica: Embrapa Agrobiologia, 2007a.
SILVA, E.E.; AZEVEDO, P.H.S.; DE-POLLI, H. Determinação do carbono da biomassa microbiana do solo (BMS-C). Comunicada Técnico 98. Seropédica: Embrapa Agrobiologia, 2007b.

SILVA, E.E.; AZEVEDO, P.H.S.; DE-POLLI, H. Determinação da respiração basal (RBS) e quociente metabólico do solo $\left(q \mathrm{CO}_{2}\right)$. Comunicada Técnico 99. Seropédica: Embrapa Agrobiologia, 2007c.

SUTER, G.W.; EROYMSON, R.A.; SAMPLE, B.E.; JONES, D.S. Ecological Risk Assessment for Contaminated Land. Boca Raton, FL, USA: Lewis Publishers, 2000.

SWARTJES, F.A.; BREURE, A.M.; BEAULIEU, M. Introduction to Ecological Risk Assessment. In: SWARTJES, F. A. (Ed.). Dealing with Contaminated Sites: From Theory towards Practical Application. Dordrecht: Springer, 2011.

SWARTJES, F.A. et al. State of the art of contaminated site management in The Netherlands: Policy framework and risk assessment tools. Science of the Total Environment, v. 427-428, p. 1-10, 2012.

USEPA - United States Environmental Protection Agency. Framework for Ecological Risk Assessment. U.S. Risk Assessment Forum, 1992.

USEPA - United States Environmental Protection Agency. Ecological Risk Assessment Guidance for Superfund: Process for Designing and Conducting Ecological Risk Assessments. Edison, NJ: USEPA, 1997.

USEPA - Unitd States Environmental Protection Agency. Guidelines for cological Risk Assessment. Washington, DC: USEPA, 1998. 